\title{
A prototype for the AMS-RICH experiment
}

\author{
G. Boudoul ${ }^{\mathrm{a}, 1}$ \\ ${ }^{a}$ Institut des Sciences Nucléaires (CNRS/IN2P3 - UJF), 53 Avenue des Martyrs, \\ 38026 Grenoble cedex, France. \\ boudoul@isn.in2p3.fr
}

\begin{abstract}
The AMS spectrometer will be installed on the International Space Station in 2005. Among other improvements over the first version of the instrument, a ring imaging Cherenkov detector (RICH) will be added and should open a new window for cosmicray physics, allowing isotope separation up to $\mathrm{A} \approx 25$ between 1 and $10 \mathrm{GeV} / \mathrm{c}$ and element identification up to $\mathrm{Z} \approx 25$ between threshold and $1 \mathrm{TeV} / \mathrm{c} /$ nucleon. It should also contribute to the high level of redundancy required for AMS and reject efficiency albedo particles. A second generation prototype has been operated for a few months : the architecture and the first results are presented.
\end{abstract}

\section{Introduction}

The AMS spectrometer [1] will be implemented on the International Space Station in 2005. The instrument will be made of a superconducting magnet which inner volume will be mapped with a tracker consisting of 8 planes of silicon microstrips with a set of detectors for particle identification placed above and below the magnet: scintillator hodoscopes, electromagnetic calorimeter (ECAL), transition radiation detector (TRD) and ring imaging Cherenkov (RICH). This contribution is devoted to a study a second generation prototype aiming at the RICH testing.

The physics capability of the RICH counter has been investigated by simulations [2]. It should provide unique informations among the AMS detectors by several respects :

1 For the AMS-RICH Collaboration: INFN Bologna, ISN Grenoble, LIP Lisbon, CIEMAT Madrid, U. Maryland, and UNAM Mexico 
- Isotopes separation up to $\mathrm{A} \approx 25$ at best, over a momentum range extending from about 1-2 GeV/c up to around $13 \mathrm{GeV} / \mathrm{c}$.

- Identification of chemical elements up to $\mathrm{Z} \approx 25$ at best, up to approximately $1 \mathrm{TeV} /$ nucleon.

- High efficiency rejection of albedo particles for momenta above the threshold, between $1 \mathrm{GeV} / \mathrm{c}$ and $3.5 \mathrm{GeV} / \mathrm{c}$ depending on the type of radiator.

The RICH counter will allow to collect a unique sample of nuclear astrophysics data with unprecedented statistical significance over a momentum range totally unexplored for the most interesting isotopes. Fig. 1 shows, as an example, the ${ }^{10} \mathrm{Be}$ to ${ }^{9} \mathrm{Be}$ ratio with 6 weeks of counting time [3]. Both the number of events and the covered energy range will dramatically improve the available data (lower left points on the plot).

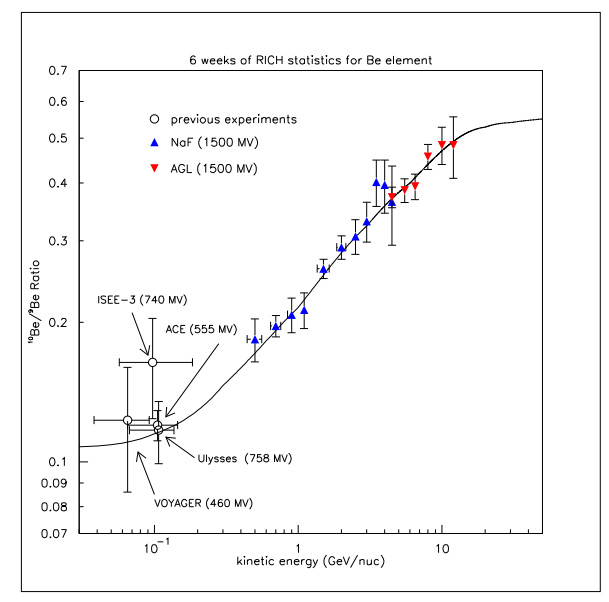

Fig. 1. Expected statistics for the ${ }^{10} \mathrm{Be}$ in 6 weeks of counting with AMS [3].

Recent works [4] have emphasized the importance of measuring cosmic nuclei spectra for: 1) Setting strong constraints on the astrophysical and cosmic ray propagation parameters of the galaxy : the diffusion coefficient normalisation and its spectral index, the halo thickness, the Alfvén velocity and the convection velocity; 2) Increasing the sensitivity to new physics search for supersymmetric particles or primordial back holes; 3) Testing for the nature of the cosmic-ray sources : supernovae, stellar flares, Wolf-Rayet stars, etc ...

\section{New prototype}

The second generation prototype has been developed by the RICH group of the AMS collaboration. It is made of one half module of the final counter and has been operated for a few months.

It is equipped with R7600-M16 PMTs from Hamamatsu Inc. Ninety-six units are used in the prototype, providing 1526 pixels. The R7600-M16 is a 16 pixels 

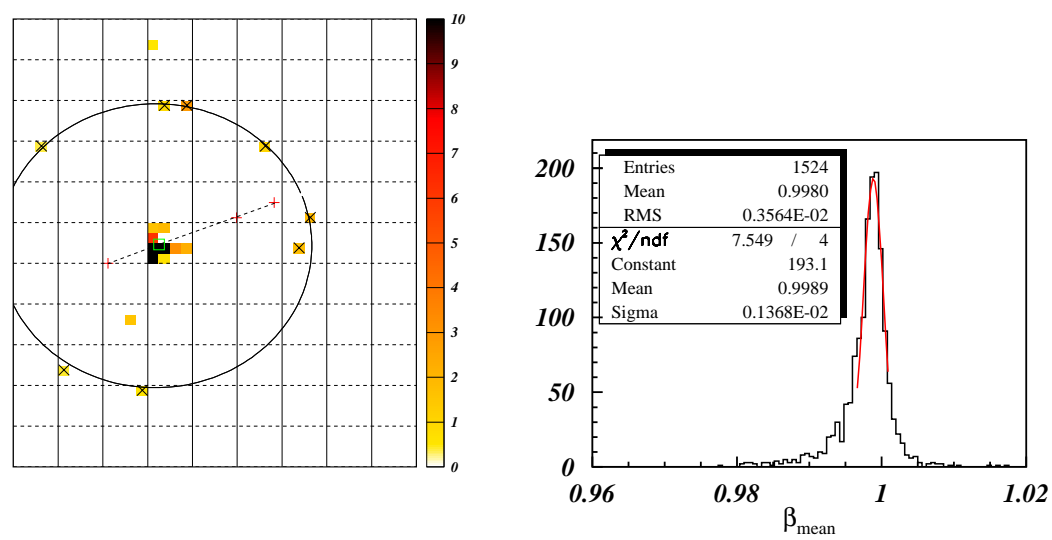

Fig. 2. Left: Cherenkov ring produced by a cosmic ray. Note the direct PMT hit (square at the center of the circle) on the reconstucted trajectory (dotted line). Right: Reconstructed velocity for $\mathrm{n}=1.03$

$\operatorname{PMT}\left(16 \times\left(4.5 \times 4.5 \mathrm{~mm}^{2}\right)\right)$ with 12 metal channel dynodes and a borosilicate glass window. The high voltage divider used is a compromise between single photoelectron resolution $\left(\frac{\sigma}{Q} \approx 0.5\right)$ and linearity. Its total resistivity was fixed at $80 \mathrm{M} \Omega$, which allows a very low power consumption and remains compatible with the expected trigger rate around $1 \mathrm{kHz}$. The front-end electronics is placed next to the PMT on a flex connector linked to the readout bus. Each PMT is equipped with solid light guides to collect the Cherenkov photons and to reduce the dead-space between photocathodes. This prototype is installed in the same instrumental setup as the previous version [5] and uses the same trigger and tracker system, which includes three NE102 plastic scintillator units, coupled with RTC-2262B PMTs, for trigger definition, and a set of three X-Y multiwire proportional chambers of $320 \times 320 \mathrm{~mm}^{2}$ with $2 \mathrm{~mm}$ wire step, and delay lines readout.

In cosmic-ray tests, the 3 available space points provide the trajectory reconstruction in the radiator with a $\sigma_{\text {track }} \approx 1.9 \mathrm{~mm}$ in both directions, by means of $\chi^{2}$ optimization procedure.

The main goals of this new generation of prototype are to validate the complex assembly procedure, check the readout electronics settings and DAQ procedure for all the output channels, investigate the PMT+electronics response dynamics, measure the counter velocity resolution, test the whole structure when submitted to vibrations, and validate the magnetic shielding efficiency.

It will also be tested with a secondary ion beam at CERN using a fragmentation target. The sample of nuclei from $\mathrm{H}$ to Fe should allow an accurate check of the charge measurement resolution of the counter. 


\section{First results with cosmic rays}

Fig 2 left gives an example of a Cherenkov ring obtained during a cosmic ray run.

The best experimental velocity resolution obtained for cosmic ray test was $\frac{\delta \beta}{\beta}=1510^{-3}$ with $\bar{n}=1.33 \mathrm{NaF}$ radiator (Cherenkov threshold around 480 $\mathrm{MeV} /$ nucleon), $\frac{\delta \beta}{\beta}=2.510^{-3}$ with $\bar{n}=1.03$ aerogel radiator as shown on

Fig 2 right, and $\frac{\delta \beta}{\beta}=3.510^{-3}$ with $\bar{n}=1.05$ (Cherenkov threshold around $3.5 \mathrm{GeV} /$ nucleon). These resolutions are limited by the multiwire chambers track reconstruction accuracy. Those results will be improved by increasing the distance between chambers. Both the electronics and the PMTs behave as expected.

The study has shown a good agreement between data and simulation and give confidence in the expected performances of the final counter.

\section{References}

[1] A. Barrau, Proc. of the "Rencontres de Moriond 2001" ; astro-ph/0103493

[2] M. Buenerd \& Z. Ren, Nucl. Inst. and Meth. in Phys. A 454 (2000) 476;

J. Casaus et al., private communication.

[3] A. Bouchet et al., Nucl. Phys. A 688 (2001) 417c

[4] Donato, F., Maurin, D., Salati, P. and al (2001), ApJ, 563, 172, in press

Barrau, A., Boudoul, G., Donato, F., Maurin, D. et al., A\&A 388, 676-687 (2002);

Yu. A. Golubkov, M. Yu. Khlopov, Phys.Rev. D62 (2000) 083505

[5] T. Thuillier et al., accepted by Nucl. Inst. and Meth. in Phys. A (2002) ; astroph/0201051 